\title{
Johannes Grössl
}

\section{Der bleibende Wert der Sterblichkeit}

\author{
- vor dem Hintergrund christlicher Anthropologie \\ und transhumanistischer Utopie
}

\begin{abstract}
Das Motiv der Vanitas, wie es im biblischen Buch Kohelet eingeführt wird, dient den Gläubigen als Erinnerung und den Nicht-Gläubigen als Warnung, dass alles Streben nach innerweltlichen Gütern ohne Gottesperspektive letztlich sinnlos ist. In diesem Beitrag werden aktuelle transhumanistische Bestrebungen nach innerweltlicher Unsterblichkeit kritisch diskutiert und mit philosophischen sowie theologischen Argumenten für einen Wert der Sterblichkeit konfrontiert. Ohne Sterblichkeit besteht die Gefahr, dass Entscheidungen an Bedeutung verlieren, Kreativität und Anpassungsfähigkeit ausgesetzt sowie moralische Verantwortung und personale Identität zerstört werden. Auch eine religiöse Erlösungs- und Auferstehungshoffnung setzt innerweltliche Vergänglichkeit und Kontingenz voraus.
\end{abstract}

\section{Überwindung der Sterblichkeit?}

Die Vergänglichkeit des Menschen wird nicht nur in verschiedenen Schriften der Bibel, sondern darauf aufbauend auch in christlicher Literatur und moderner Theologie häufig und intensiv thematisiert. $\mathrm{Zu}$ den einflussreichen Motiven gehört dabei die Sündenfall-Erzählung, die erklären soll, wie Leid und Tod in die Welt kamen, sowie das Motiv der Vanitas, wie es in der alttestamentlichen Weisheitsliteratur eingeführt wurde: Weltliche Güter sind letztlich ,Nichtigkeit", weswegen man die Hoffnung auf die ewigen Dinge setzen solle. Als Teil einer solchen Hoffnung auf das Ewige gehört das Versprechen von Unsterblichkeit zum Kernbestand von Religion. ${ }^{1}$ Dieses Versprechen wird aber in letzter Zeit auch von ganz anderer Seite ausgesprochen, und zwar auf der Grundlage eines naturalistischen Weltbildes. Mithilfe der Wissenschaft soll der Mensch von seinen

1 Gerade das Christentum lässt sich als eine transhumanistische Religion bezeichnen, weil es nach einer Transformation zur Perfektion jedes einzelnen Individuums strebt. Vgl. Ronald ColeTurner. „Von der Theologie zum Transhumanismus und zurück“. Designobjekt Mensch. Die Agenda des Transhumanismus auf dem Prüfstand. Hrsg. von Benedikt Göcke und Frank Meier-Hamidi. Freiburg: Herder, 2018. 293-308, S. 293-297.

Ә Open Access. ( 2021 Johannes Grössl, publiziert von De Gruyter. (c) BY-NC-ND Dieses Werk ist lizensiert unter einer Creative Commons Namensnennung-Nicht-kommerziell-Keine Bearbeitung 4.0 International Lizenz. 
natürlichen Begrenzungen befreit, vielleicht sogar unsterblich gemacht werden. Der Begriff ,Transhumanismus‘ beschreibt eine heterogene, wachsende globale Bewegung, die dieses Ziel durch die Entwicklung verschiedener Technologien erreichen möchte. Doch hierbei stellt sich die Frage, ob es sinnvoll ist, nach einer Überwindung der (innerweltlichen) Sterblichkeit zu streben, oder ob Sterblichkeit nicht auch etwas Wertvolles ist, für dessen Aufrechterhaltung es gute Gründe gibt.

Für eine philosophische Diskussion darüber, ob Sterblichkeit ein Gut oder ein Übel darstellt, müssen zunächst die möglichen Folgen einer Überwindung der Sterblichkeit für menschliche Personen und Gesellschaften evaluiert werden. Dazu ist es auch notwendig, darüber nachzudenken, welche Auswirkungen auf das Subjekt Sterblichkeit mit sich bringt: Welchen Einfluss hat das Wissen, dass wir einmal sterben müssen, auf unser Verständnis des Menschseins, auf unseren Charakter, auf unsere Lebensführung? In diesem Beitrag werden zunächst die gesellschaftlichen Herausforderungen, welche die transhumanistische Agenda in den nächsten Jahrzehnten mit sich bringen wird, dargelegt. Anschließend werden sowohl philosophische als auch theologische Argumente für und gegen die transhumanistischen Bestrebungen vorgestellt und kritisch diskutiert. Abschließend werden einige biblische und kulturgeschichtliche Motive aufgezeigt, die eine Würdigung der menschlichen Sterblichkeit unterstützen.

\section{Gesellschaftliche Herausforderung und Zielsetzung}

Es ist absehbar, dass innerhalb der nächsten Jahrzehnte immer mehr Technologien auf den Markt kommen werden, mit denen die Lebenserwartung derjenigen, die sich diese leisten können, deutlich verlängert werden kann. Hierzu gehören sowohl medizinische, gentechnische als auch kybernetische Maßnahmen. Blickt man überdies auf das nächste Jahrhundert, könnten womöglich Technologien entwickelt werden, mit denen eine Quasi-Unsterblichkeit erreicht werden kann, z. B. durch Körpertransplantationen, Bewusstseins-Uploads auf Computern oder durch sukzessiven Austausch von biologischen durch kybernetische Körperteile. ${ }^{2}$ Dabei stehen wir jedoch vor zwei ethischen Herausforderungen. Einerseits: Welche technischen Verbesserungen des Menschen sollen moralisch und rechtlich erlaubt sein und welche nicht? Andererseits: Wenn es absehbar ist, dass diese Ent-

2 Die meisten heutigen Transhumanistinnen und Transhumanisten streben nicht Unsterblichkeit an, sondern eine deutliche Verlängerung von Lebensqualität und -dauer. Vgl. Stefan Sorgner. „Was wollen Transhumanisten?“ Designobjekt Mensch. Die Agenda des Transhumanismus auf dem Prüfstand. Hrsg. von Benedikt Göcke und Frank Meier-Hamidi. Freiburg: Herder, 2018. 153-180, S. 157. 
wicklung nicht verhindert werden kann (ohne massiv in individuelle Freiheitsrechte einzugreifen oder globale Hegemonie zu betreiben), welche Maßnahmen sollten wir ergreifen, um diese Entwicklung zu begleiten und bestimmte Risiken, die damit verbunden sind, zu minimieren? Es ist in unserem aktuellen politischen und wirtschaftlichen System diesbezüglich auch absehbar, dass entsprechende Technologien zunächst nur dem wohlhabenden Teil der Bevölkerung zur Verfügung stehen werden. Dies würde mit hoher Wahrscheinlichkeit zu einer Aufspaltung der (regionalen oder globalen) Gesellschaft in zwei Klassen führen. Das heißt: Auch wenn die Überwindung von Sterblichkeit bzw. eine Verlängerung des menschlichen Lebens grundsätzlich erstrebenswert scheint, könnte argumentiert werden, dass die Nebeneffekte auf dem Weg zur Erreichung dieses Ziels viele Probleme und Leid mit sich bringen würden, sodass es aus diesem Grund vorerst nicht angestrebt werden sollte.

Doch neben diesen praktischen Gründen - die nur temporär Gültigkeit haben und die man durch entsprechende politische Maßnahmen deutlich abschwächen könnte - gibt es auch einige grundsätzliche Bedenken in Bezug auf die transhumanistische Agenda. Diese kommen primär von Seiten bestimmter anthropologischer Traditionen, die mit einer philosophischen Einschätzung der menschlichen Natur einhergehen. Sie geben also mögliche Antworten auf die Frage, was den Menschen in seinem Wesen ausmacht. Es gibt Anthropologien, die mit einer transhumanistischen Agenda weitgehend kompatibel sind: In transhumanistischer Literatur wird häufig auf Giovanni Pico della Mirandola verwiesen, Vordenker des Humanismus im 15. Jahrhundert, der dafür geworben hat, dass der Mensch sich nicht einem vorgegebenen (anthropologischen) Ideal unterwerfen sollte, sondern es gerade sein Wesen ausmacht, dass er seine Natur selbst bestimmen kann und soll. ${ }^{3}$ Auch Friedrich Nietzsches Theorie des Übermenschen wird häufig als Prototyp transhumanistischer Bestrebungen angesehen. ${ }^{4}$ Im 19. und 20. Jahrhundert lässt sich vor allem der Utilitarismus als transhumanismuskompatible Bewegung ausmachen: Wenn es grundlegendes Ziel der menschlichen Existenz ist, Leid zu minimieren und Lust zu maximieren, folgt daraus, dass es ceteris paribus geboten ist, das menschliche Leben so weit wie möglich zu verlängern. ${ }^{5}$ Doch auch hier lässt sich hinterfragen, ob eine fast unbegrenzte Lebensdauer unter Einbeziehung aller Faktoren tatsächlich die Lebensqualität verbessern und alle Werte, welche die menschliche Existenz ausmachen, erhalten würde. Um dies einschätzen zu können, benötigt es

3 Vgl. Otto Hansmann. „Begriff und Geschichte des Transhumanismus“. Designobjekt Mensch. Die Agenda des Transhumanismus auf dem Prüfstand. Hrsg. von Benedikt Göcke und Frank Meier-Hamidi. Freiburg: Herder, 2018. 25-51, S. 36-37.

4 Vgl. ebd., S. 38-41.

5 Vgl. ebd., S. 41-43. 
eine spezifischere Diskussion über die menschliche Natur, also eine Anthropologie, die neben maximaler Gestaltungsfähigkeit auch andere wesentliche Eigenschaften oder zu erstrebende Güter berücksichtigt.

\section{Philosophische Bewertung der Sterblichkeit}

Sterblichkeit wird in ethischen Theorien meist als zu vermeidendes Übel angesehen. Allerdings gilt dies selbst in einer utilitaristischen Ethik nicht uneingeschränkt; dort können Sterblichkeit und der Tod selbst sogar als etwas Gutes angesehen werden, und zwar in Fällen, in denen zukünftiges Leiden verhindert werden kann. Deswegen sprechen sich Anhängerinnen und Anhänger des Utilitarismus in der Regel auch für die moralische und legale Erlaubnis von Sterbehilfe oder assistiertem Suizid von Schwerkranken aus. ${ }^{6}$ In vielen deontologischen Ethiken gilt hingegen der Erhalt des Lebens als oberster Wert, häufig sogar noch deutlich stärker als beim Utilitarismus, da dieser absolute Wert nicht von äußeren Bedingungen oder zukünftigen Entwicklungen abhängig ist. In vielen kantisch sowie naturrechtlich-christlich geprägten Ethikkonzeptionen gilt deswegen Sterbehilfe unter allen Umständen als verwerflich. ${ }^{7}$ Der Transhumanismus geht allerdings davon aus, dass nicht nur die Lebensdauer, sondern auch die Lebensqualität langfristig erhalten werden kann, weswegen auch eine utilitaristische Nutzen-Kosten-Abwägung immer auf der Seite des Lebenserhalts stehen sollte. ${ }^{8}$

Aus rein philosophischer Sicht, d. h. unter Absehung von religiösen Prämissen bezüglich der Natur des Menschen, lassen sich drei gewichtige Einwände gegen eine unbegrenzte Verlängerung des menschlichen Lebens einbringen, selbst wenn die Lebensqualität (im Sinne von Abwesenheit physischen Leidens) aufrechterhalten werden kann: (1) Kontingenzerfahrungen motivieren uns dazu, die Begrenzun-

\footnotetext{
6 Vgl. Dieter Birnbacher. „Sterbehilfe - Eine philosophische Sicht“. Aktive und passive Sterbehilfe. Medizinische, rechtswissenschaftliche und philosophische Aspekte. Hrsg. von Felix Thiele. München: Fink, 2005. 31-42.

7 Vgl. Eberhard Schockenhoff. Ethik des Lebens. Grundlagen und neue Herausforderungen. Freiburg: Herder, 2016. 537-559. Bezüglich der säkularen, sich auf Immanuel Kant berufenden Ethik sei erwähnt, dass, während eine Ablehnung jeglicher Form des Suizids bei Kant selbst eindeutig zu erkennen ist, es kontrovers diskutiert wird, ob eine solche Ablehnung notwendigerweise aus einer kantischen Ethik folgt. Vgl. Ian Brassington. „Killing People: What Kant Could Have Said about Suicide and Euthanasia but Did not“. Journal of Medical Ethics 32.10 (2006): 571-574.

8 Bereits die griechische Mythologie kennt die Figur des Tithonius (z. B. Homers Hymnus an Aphrodite), der auf eigenen Wunsch von Zeus zwar Unsterblichkeit erhielt, allerdings vergaß, auch um ewige Jugend zu bitten. Da seine Lebensqualität aufgrund zunehmenden Alters drastisch gemindert wurde, wünschte er sich schließlich den Tod.
} 
gen der menschlichen Natur überwinden zu wollen; dieses Streben, die eigene Begrenzung zu überwinden (und dabei die eigene Identität $\mathrm{zu}$ bewahren), stiftet Sinn. (2) Die Gewichtigkeit von Entscheidungen nimmt mit zunehmender verfügbarer Zeit ab, diese zu revidieren oder alternative Optionen später zu realisieren. Sterblichkeit verleiht unseren Lebensentscheidungen Bedeutung. (3) Neue Paradigmen und Denkschemata setzen sich meist nur durch, da die ,alte Generation“ ausstirbt. Dies liegt vor allem daran, dass das Innovationspotenzial und die Anpassungsfähigkeit bei eingefahrenen Strukturen immer geringer werden.

Gerade das erste Argument ist für viele Menschen mit Verweis auf eigene Erfahrungen und Zukunftsvorstellungen leicht nachvollziehbar und wird auch von diverser fiktionaler Literatur sowie von Filmen gestützt. ${ }^{9}$ Der Philosoph Tony Coady argumentiert sogar, dass diese Minderung der Bedeutung von Entscheidungen auch unsere Motivation, irgendetwas zu erreichen, zerstört; ein großer Teil unseres Strebens sei nämlich ein Streben danach, natürliche Begrenzungen zu überwinden:

Unser Streben nach dem guten Leben findet vor dem Hintergrund unserer natürlichen Grenzen statt. Wir können diese Grenzen im Prozess des Strebens nach größeren menschlichen Errungenschaften verschieben und verändern, so wie der Sportler über das hinausgeht, was er für möglich gehalten hat, indem er sich anstrengt, die bestehende Begrenzung des menschlichen Körpers zu überwinden. Aber wir brauchen die weit gefasste Idee einer solchen Begrenzung, um dieser Überwindungsbemühung [surpassing performance] einen Sinn zu geben. ${ }^{10}$

Bernard Williams hat den ,Einwand der Langeweile‘ gegen die Unsterblichkeit erhoben, der auf ähnlichen Intuitionen basiert. Williams stellt ein Dilemma dar: Angenommen, Menschen können mit der Zeit ihre Gewohnheiten in einem solchen Maße festigen, dass sie irgendwann nicht mehr die Freiheit haben, diese zu ändern. Wenn eine solche irreversible Charakterformung möglich wäre, würde „das Spektrum der möglichen Ziele und Projekte einer Person eingegrenzt und über einen ausreichend langen Zeitraum erschöpft sein, sodass das Ergebnis eine

9 Dass Unsterblichkeit zu Apathie oder gar Depression führen kann, wird z. B. in Mary Shelleys Kurzgeschichte „The Mortal Immortal“ (1833) oder in Jorge Borges „El Inmortal“ (1947) vorgeführt. Auch in Science-Fiction-Literatur sowie -Serien werden negative psychologische Folgen von Unsterblichkeit aufgezeigt; vgl. z. B. Star Trek Voyager, Staffel 2, Episode 18 („Death Wish“; 13:35-13:45): „[W]e sacrificed many things along the way, not just manners, but mortality, the sense of purpose, and a desire for change, and a capacity to grow.“

10 Cecil Anthony John [Tony] Coady. „Playing God“. Human Enhancement. Hrsg. von Julian Savulescu und Nick Bostrom. New York, NY: Oxford University Press, 2009. 155-180, S. 178 [Übers. des Verf.]. 
endlose Langeweile wäre“11. Möglicherweise würde ein solcher Mangel an Kontingenz das moderne Subjekt sogar ganz zerstören, weil dadurch, so argumentiert Katharina Klöcker, die Individualität menschlicher Personen vernichtet werden würde. ${ }^{12}$ Wenn sich hingegen die Charaktere immer wieder verändern könnten, so Williams, würde die Veränderung über einen sehr langen Zeitraum zu einer völlig anderen Person führen, was das zentrale Ziel der Unsterblichkeit verletzen würde: die eigene Identität $\mathrm{zu}$ bewahren. ${ }^{13}$ Daher sei eine sterbliche Existenz in beiden Fällen der Unsterblichkeit vorzuziehen.

Auch das zweite Argument ist im Hinblick auf die Ausbildung von Identität verständlich. Wenn man unendlich viel Zeit hat, dann braucht man sich beispielsweise nicht zu entscheiden, welches Fach man studiert, welche Karriere man anstrebt oder welches Instrument man lernt. Man entscheidet sich nur noch, welche Option man zeitlich vorzieht; alles andere lässt sich in der Zukunft nachholen. Aber: Entscheidungen werden erst primär aufgrund ihrer Exklusivität und Irreversibilität als identitätsbildend, sinnstiftend und bedeutsam erachtet. ${ }^{14}$ Die Tatsache, dass unsere Zeit begrenzt ist, spielt daher eine wichtige Rolle, um Entscheidungen Bedeutung zuzumessen. ${ }^{15}$ Jeff Malpas argumentiert, dass ein menschliches Leben

11 Roman Altshuler. „Immortality, Identity, and Desirability“. Immortality and the Philosophy of Death. Hrsg. von Michael Cholbi. Lanham, MD: Rowman \& Littlefield, 2015. 191-203, S. 194 [Übers. des Verf.]; vgl. auch Bernard Williams. „The Makropoulus Case: Reflections on the Tedium of Immortality“. Problems of the Self: Philosophical Papers 1956-1972. Cambridge: Cambridge University Press, 1973. 82-100.

12 Vgl. Katharina Klöcker. „Zur ethischen Diskussion um Enhancement. Eine kritische Anmerkung zum Transhumanismus aus theologisch-ethischer Perspektive“. Designobjekt Mensch. Die Agenda des Transhumanismus auf dem Prüfstand. Hrsg. von Benedikt Göcke und Frank MeierHamidi. Freiburg: Herder, 2018. 309-338, S. 334. Für eine kritische Diskussion dieser Behauptung vgl. z. B. Aaron Smuts. „Immortality and Significance“. Philosophy \& Literature 35.1 (2011): 134-149.

13 Vgl. Altshuler 2015 (Anm. 11), S. 195; Michael Cholbi. „Immortality and the Exhaustibility of Value“. Immortality and the Philosophy of Death. Hrsg. von dems. Lanham: Rowman \& Littlefield, 2015. 221-236, S. 221: „[...] that the individual we would thereby become would become so different from us that we could not recognize their good as ours".

14 Vgl. Cholbi 2015 (Anm. 13), S. 231. Cholbi fasst diese Überlegungen zusammen, indem er sagt, dass Sterblichkeit die ,Bedeutung des Handelns“ erhöhe: „The second reason, then, to prefer mortal life to immortal life is that mortality makes agency matter more. Our agency has greater shape and efficacy in a mortal life; how our life turns out depends much more on what we choopse and do.“

15 Vgl. Samuel Scheffler. Death and the Afterlife. New York, NY: Oxford University Press, 2013, S. 99-100. Dies ist auch das zentrale Argument in Odo Marquard. „Finalisierung und Mortalität“. Das Ende. Figuren einer Denkform. Hrsg. von Karlheinz Stierle und Rainer Warning. München: Fink, 1996. 467-475. 
ein Gefühl des Abschlusses, eine Möglichkeit des Todes haben muss, denn die einzelnen Projekte dieses Lebens beziehen ihre Relevanz aus dem Sinn des Lebens als Ganzes. ${ }^{16}$ Gerade das Wissen darum, dass wir viele Entscheidungen nicht mehr rückgängig machen können, verleiht ihnen Gewicht. ${ }^{17}$

Eine fast unendliche Anzahl von Optionen bedeutet jedoch keine Zerstörung der Kontingenz. Solange unser freier Wille erhalten bleibt, können wir - auch als Unsterbliche - wählen, was für Menschen wir sein wollen: Wir können moralisch signifikante Entscheidungen treffen, Gewohnheiten (Tugenden oder Laster) ausprägen und unseren Charakter - möglicherweise irreversibel - in die eine oder andere Richtung formen. Unsterblichkeit zerstört nicht die Kontingenz; sie ermöglicht sogar mehr Optionen. Sie verleiht manchen Entscheidungen mehr Gewicht, wie z. B. dem Eingehen einer unlösbaren Ehe oder der Bereitschaft, das eigene Leben für einen guten $\mathrm{Zweck}$ zu riskieren. ${ }^{18}$

Darüber hinaus gäbe es selbst für eine unsterbliche Person Tatsachen, über die sie keine Macht hat, sowohl über ihre eigene Existenz als auch, mehr noch, über die Existenz und Individualität anderer. Diese Begrenzung von Allmacht wird nicht nur philosophisch und theologisch, sondern auch in künstlerischen Kontexten reflektiert: Im fiktiven Star-Trek-Universum etwa wird eine transhumanistische Spezies eingeführt, die sich selbst als allmächtig ansieht: das Q-Kontinuum. Deren Mitglieder sind nicht nur unsterblich, sie können spielend leicht sogar Raum und Zeit beliebig manipulieren. Doch man erfährt schließlich, dass auch diese Allmacht an ihre Grenzen stößt. So stellt sich im Verlauf des Handlungsstrangs einer Episode heraus, dass auch ein Mitglied des Q-Kontinuums eine andere Person nicht dazu bringen kann, sie zu lieben. ${ }^{19}$ Unsterblichkeit

16 Vgl. Altshuler 2015 (Anm. 11), S. 194.

17 Vgl. Richard Swinburne. The Existence of God. Oxford: Oxford University Press, 2004, S. 229: „[A] world with natural death would be a world in which an agent's own contribution would have a seriousness about it because it would be irreversible by the agent. If I spend all my seventy years doing harm, there is no time left for me to undo it. But, if I live for ever, then, whatever harm I do, I can always undo it. It is good that what people do should matter, and their actions matter more if they have only a limited time in which to reverse them.“

18 Vgl. ebd.; siehe auch die dortige Aussage: „The ultimate sacrifice is the sacrifice of oneself, and that would not be possible in a world without death."

19 Vgl. Star Trek: The Next Generation, Staffel 6, Episode 6 („True Q“). Das Motiv, dass Allmacht mit Liebe unvereinbar ist, findet sich bereits bei Kierkegaard und wird im modernen christlichen Kontext meist verwendet, um die Notwendigkeit von Kenosis und Inkarnation zu begründen. Vgl. Søren Kierkegaard. „Eine literarische Anzeige“. Gesammelte Werke 17. Übers. von Emanuel Hirsch. Düsseldorf: Eugen Diederichs, 1954, 124-125; ders. Philosophische Brocken. Übers. und hrsg. von Liselotte Richter. Hamburg: Europäische Verlagsanstalt, 1992, 27-33. 
impliziert nicht automatisch Allmacht, und selbst wenn sie es täte: Letztere unterliegt logischen Grenzen und wird daher auch durch den freien Willen anderer eingeschränkt. ${ }^{20}$ Dementsprechend bedroht Unsterblichkeit unsere individuelle Autonomie nicht radikal, solange es Kontingenz und die Möglichkeit irreversibler Handlungen gibt. Wenn man nur eine Quasi-Unsterblichkeit anstrebt, mit der weiter bestehenden Möglichkeit, sich selbst und anderen zu schaden, wird es auch weiterhin möglich sein, zahlreiche sinnvolle (gute wie schlechte) Entscheidungen zu treffen.

Das dritte Argument ist nachvollziehbar, wenn man sich eine Gesellschaft vorstellt, bei der es keinen Grund mehr für (und sogar zunehmend Gründe gegen ${ }^{21}$ ) Fortpflanzung gibt. Dort ist nicht nur die moralische Verantwortung des Individuums deutlich geringer, da alles wegefällt, was mit der Erziehung von Nachkommen und der Weitergabe der Kultur und von Werten verbunden ist. Stattdessen besteht die Gefahr, dass die gesamte Gesellschaft letztendlich in eine Stagnation verfällt. Die meisten Menschen bleiben mit zunehmendem Alter in bestimmten Denkmustern und Gewohnheiten stecken - ganz unabhängig von ihrem Bildungshintergrund. Der Wissenschaftsphilosoph Thomas Kuhn verteidigt die These, dass neue Paradigmen in der Wissenschaft in der Regel nicht über andere triumphieren, indem sie die Befürworter des alten Leitbildes, in das sie selbst Mühe und Zeit investiert haben, überzeugen, sondern es vielmehr die neuen kreativen Köpfe sind, die einen Paradigmenwechsel in der Wissenschaft bewirken. ${ }^{22}$ In seinem einflussreichen Werk Structure of Scientific Revolution zitiert Kuhn den Physiker Max Planck, der es nicht einmal geschafft hat, seinen Freund Albert Einstein von einigen Implikationen der Quantentheorie zu überzeugen. Planck kommentiert hierzu:

20 So wie ein allmächtiges Wesen keinen eckigen Kreis erschaffen kann, ist es logisch auch nicht möglich, dass ein allmächtiges Wesen freie Wesen erschafft und gleichzeitig die Entscheidungen dieser Wesen determiniert. Vgl. Johannes Grössl. [Art.] „Allmacht“. Theologische Grundbegriffe. Ein Handbuch. Hrsg. von Cornelia Dockter, Martin Dürnberger und Aaron Langenfeld. Paderborn: utb/Schöningh, 2020.18-19.

21 Vgl. Brent Waters. „Flesh Made Data. The Posthuman Project in Light of the Incarnation“. Religion and Transhumanism. The Unknown Future of Human Enhancement. Hrsg. von Calvin Mercer und Tracy J. Trothen. Santa Barbara, CA: Praeger, 2015. 291-302, S. 297: „If one is endeavoring to live for as long as possible, and perhaps for forever, then future generations are not only unnecessary, but may prove to be another external constraint imposed on the will or, even worse, unwanted competition.“

22 Vgl. Thomas Kuhn. Structure of Scientific Revolution. 2. Aufl. Chicago, IL: University of Chicago Press, 1970, S. 152: „Conversions will occur a few at a time until, after the last holdouts have died, the whole profession will again be practicing under a single, but now a different, paradigm.“ 
Eine neue wissenschaftliche Wahrheit pflegt sich nicht in der Weise durchzusetzen, daß ihre Gegner überzeugt werden und sich als belehrt erklären, sondern vielmehr dadurch, daß ihre Gegner allmählich aussterben und daß die heranwachsende Generation von vornherein mit der Wahrheit vertraut gemacht ist. ${ }^{23}$

Eine Überwindung der Sterblichkeit könnte also das Potenzial einer Gesellschaft, Innovationen hervorzubringen, deutlich senken. Auch hier ist zur Illustration das bereits erwähnte fiktive Q-Kontinuum im Star-Trek-Universum anzuführen: Es hat in seiner Entwicklung einen Zustand erreicht, in dem keine Veränderung mehr stattfindet und jeder mit seinem Daseins- und Denkzustand zufrieden ist. Erst als ein Q für sein Recht auf Selbstmord plädierte, die Gesellschaft davon überzeugte, ihm seine Unsterblichkeit zu nehmen, und sich schließlich selbst tötete, kam es zu Neuerungen: Die Gesellschaft passte sich an neue Umstände an, vor allem an die Entwicklung anderer Spezies. Sie konnte nun wahrnehmen, dass sich dort Charaktereigenschaften herausgebildet hatten, die den ihren überlegen sein könnten. Da dieser Wandel die Gesellschaft zerrüttete, nahm ein Q die Gewohnheit der Fortpflanzung erneut auf, um das Kontinuum wieder zu stabilisieren - was schließlich erfolgreich war. ${ }^{24}$

\section{Positive Bewertung der Sterblichkeit in der Theologie}

In der christlichen Tradition gelten nach der Erzählung des Sündenfalls, die im alttestamentlichen Buch Genesis unmittelbar der zweiten (älteren) Schöpfungsgeschichte folgt, sowohl Schmerz als auch Sterblichkeit als Folge des Sündenfalls (vgl. Gen 2,16-17 und 3,16-19). Eine genaue Lektüre bestätigt diese traditionelle Interpretation allerdings nicht; Unsterblichkeit galt im Paradies als prinzipiell erreichbar (symbolisiert durch den Baum des Lebens, vgl. Gen 3,22), aber noch nicht verwirklicht - möglicherweise wurde sie noch nicht einmal erstrebt. ${ }^{25}$ Weil eine historische Interpretation dieser Erzählung aufgrund der Plausibilität der damit unvereinbaren Evolutionstheorie wissenschaftlich nicht mehr haltbar ist, wird in der Dogmatik meist eine rein anthropologische Erklärung herangezogen: Gott habe ursprünglich eine Welt intendiert, in der es kein Leiden und keinen Tod gibt, und er habe einen Menschen erschaffen wollen, der für eine

23 Max Planck. Wissenschaftliche Selbstbiographie. Halle/Saale: Deutsche Akademie der Naturforscher Leopoldina, 1990, S. 15.

24 Vgl. Star Trek: The Next Generation, Staffel 1, Episode 1 („Encounter at Farpoint“); Star Trek: Voyager, Staffel 2, Episode 18 („Death Wish“); Staffel 3, Episode 11 („The Q and the Grey“).

25 Vgl. Gabriele Spira. Paradies und Sündenfall. Stoffe und Motive der Genesis 3-Rezeption von Tertullian bis Ambrosius. Frankfurt a. M.: Peter Lang, 2014, S. 30-31, 34-35 und 272-273. 
Gemeinschaft mit Gott und damit zum ewigen Leben fähig sei. ${ }^{26}$ Der Grund dafür, dass diese von Gott gewollte Welt nicht unmittelbar Realität wurde, lässt sich nicht klar benennen; jedenfalls kann man diesen nicht bei der freien Entscheidung ursprünglicher Menschen suchen. ${ }^{27}$

Wenn die Paradieserzählung keine historische Genese beschreibt, kann sie uns dennoch etwas über die menschliche Natur mitteilen: Der Mensch existiert nicht in dem Zustand, der von Gott intendiert ist. Sterblichkeit und Leiden sind Defizite, von denen Gott möchte, dass sie überwunden werden. Der Mensch ist von Gott erschaffen, um in eine ewige Gemeinschaft mit seinem Schöpfer einzutreten. Die christliche Eschatologie zielt somit eindeutig auf ein ewiges Leben als Fortbestand der personalen Individualität. Dieses wird aber mehrheitlich nicht als bloße zeitliche Fortdauer des irdischen Lebens angesehen, sondern als Existenz in einer ,neuen Schöpfung، ${ }^{28}$ Transhumanistische Utopien werden deshalb oft abgelehnt oder zumindest sehr kritisch betrachtet, weil sie die innerweltliche Unsterblichkeit, die sie versprechen, aufgrund physikalisch unüberwindbarer Begrenzungen niemals einhalten können. ${ }^{29}$ Da sich naturwissenschaftlich orientierte Anhängerinnen und Anhänger des Transhumanismus dieser Restriktionen bewusst sind, propagieren sie meist keine Unsterblichkeit, sondern nur eine extreme Verlängerung der Lebensspanne. ${ }^{30}$ So könnte man meinen, dass transhumanistische Agenda und der Glaube an ein ewiges Leben überhaupt nicht in Konkurrenz zueinander stehen.

26 Vgl. Ian McFarland. „The Fall and Sin“. The Oxford Handbook of Systematic Theology. Hrsg. von John Webster, Kathryn Tanner und Iain Torrance. New York, NY: Oxford University Press, 2007. 140-159.

27 Einige Interpretationen postulieren sogar, dass der Sündenfall die Erlangung einer bestimmten Reife (maturity of humanity) symbolisiert und damit letztlich von Gott gewollt ist. Der Mensch erlange durch den Fall nicht Sterblichkeit, sondern Bewusstsein über seine eigene Vergänglichkeit. Vgl. Thomas Krüger, „Sündenfall? Überlegungen zur theologischen Bedeutung der Paradiesgeschichte“. Beyond Eden. The Biblical Story of Paradise (Genesis 2-3) and Its Reception History. Hrsg. von Konrad Schmid und Christoph Riedweg. Tübingen: Mohr Siebeck, 2008. 98-109, S. 101.

28 Vgl. Matthias Reményi. Auferstehung denken. Anwege, Grenzen und Modelle personaleschatologischer Theoriebildung. Freiburg: Herder, 2016, S. 401-402 und 626-627.

29 So besagt u. a. der Zweite Hauptsatz der Thermodynamik, dass die Entropie (Grad der Unordnung) eines geschlossenen Systems niemals abnimmt. Da Leben als Ordnungsprozess nur aufrechterhalten werden kann, wenn woanders im Universum eine Entropiezunahme geschieht (v. a. durch Kernfusion), ist es absehbar, dass irgendwann kein Leben mehr im Universum möglich sein wird. Man spricht auch davon, dass das Universum den ,Wärmetod' sterben wird, d. h. ein thermisches Gleichgewicht ohne komplexe Strukturen erreicht. Vgl. zur Entropie in den Romanen Daniel Kehlmanns den Beitrag von Verena Russlies in diesem Band.

30 Vgl. Sorgner 2018 (Anm. 2), S. 157. 
Allerdings gehen alle monotheistischen Religionen davon aus, dass für die ewige Gemeinschaft mit Gott ein gewisser Charakterbildungsprozess notwendig ist. Im Christentum nimmt man an, Gott will, dass alle Menschen gerettet werden (vgl. 1 Tim 2,4); dies schließt aber nicht aus, dass es auch für Gott unmöglich sein kann, bestimmte Menschen in Gemeinschaft zu sich zu berufen. ${ }^{31}$ Optimistisch kann man dafür votieren, dass alle Menschen, die ihren Charakter nicht irreversibel zum Schlechten geformt haben, von Gott nach ihrem Tod transformiert werden können, damit sie zu einer solchen Gemeinschaft fähig sind. Genau hier kann aber die christliche Kritik am Transhumanismus ansetzen: Unsterblichkeit könnte die Wahrscheinlichkeit erhöhen, dass ein einzelner Mensch seinen Charakter irreversibel zum Bösen formen kann. Im Umkehrschluss hieße dies, dass Sterblichkeit das Risiko auf ewige Verdammnis senkt. Auch innerweltlich hat die Einschränkung des Bösen durch Sterblichkeit Vorteile; denn es wird auch das Ausmaß an Bösem reduziert, welches ein einzelner Mensch in die Welt bringen kann:

[I]m Interesse des potenziell Leidenden muss es eine Grenze für das Leid geben, das ein Handelnder einem anderen zufügen kann. Es wäre - so glaube ich, dass wir alle urteilen würden - moralisch falsch, wenn ein sehr mächtiges Wesen einer Person grenzenlose Macht gäbe, einen anderen zu verletzen. Jemandem die Macht zu geben, zu töten, bedeutet, eine enorme Macht zu geben, die sich qualitativ von anderen Fähigkeiten unterscheidet. [...] Ein Gott, der dem Ausmaß des Leidens, das ein Geschöpf erleiden kann, keine Grenze setzt, [...] wäre kein guter Gott. Es muss Grenzen für die Intensität des Leidens und für die Dauer des Leidens geben. Ein natürlicher Tod nach einer kleinen endlichen Anzahl von Jahren stellt die Grenze der Leidensperiode dar. Es ist eine Grenze für die Macht eines Handelnden über eine andere Person. ${ }^{32}$

Der Religionsphilosoph Richard Swinburne argumentiert hier, dass durch die menschliche Sterblichkeit das Ausmaß des Theodizee-Problems gemildert wird: Bei diesem, unter anderem auch durch Albert Camus' Die Pest (1947) in der Literatur verhandelten Problem, wird reflektiert, ob die Existenz eines allmächtigen, allgütigen Gottes mit dem zu beobachtenden Übel in der Welt vereinbar ist.

Das Wissen um die eigene Begrenztheit und Endlichkeit kann auch konkret die Ausbildung bestimmter Tugenden fördern und vor der Ausbildung von Lastern schützen. So gilt Hochmut (superbia) in christlicher Tradition als Todsünde, was bedeutet, dass sie (zumindest in ihrer irreversibel ausgeprägten bzw. nicht bereuten Form) eine ewige Gemeinschaft mit Gott verunmöglicht. Wer sich nur selten

31 Vgl. Jerry Walls. „One Hell of a Problem for Christian Compatibilists“. Free Will and Theism. Connections, Contingencies, and Concerns. Hrsg. von Kevin Timpe und Daniel Speak. Oxford: Oxford University Press, 2016. 79-98.

32 Swinburne 2004 (Anm. 17), S. 229-230 [Übers. des Verf.]. 
mit eigenen Begrenzungen auseinandersetzen muss und keine Angst vor dem eigenen Tod hat, ist in größerer Gefahr, Hochmut auszubilden. ${ }^{33}$ Außerdem fallen in der transhumanistischen Utopie viele Gelegenheiten weg, bei denen andere Menschen unsere Hilfe benötigen und auf uns existenziell angewiesen sind. Aus christlicher Sicht kann das Schöpfungspotenzial einzelner Individuen dadurch realisiert werden, dass neue Lebewesen zur Existenz gebracht sowie zu autonomen Personen erzogen werden - Sterblichkeit ermöglicht und fördert dieses Potenzial.

\section{Biblische und kulturgeschichtliche Motive}

Das alttestamentliche Buch Kohelet, dem auch das Vanitas-Motiv entstammt, erinnert an die Vergänglichkeit des Menschen und die falsche Sinnperspektive durch innerweltliche Güter: „Und siehe, das alles war Nichtigkeit [הבל, vanitas] und ein Haschen nach Wind. Also gibt es keinen Gewinn unter der Sonne.“ (Koh 2,11 ${ }^{\mathrm{ELB}}$ ) Selbst ein lang andauerndes Leben in Wohlstand sei ohne die Erfüllung ewigen Glücks sinnlos (vgl. Koh 6). Diese Vorstellung, dass es ohne eschatologische Perspektive nicht möglich ist, dem Leben nachhaltigen Sinn zu verleihen, wird in der christlichen Tradition häufig aufgegriffen. Von Augustinus stammt der berühmte, an Gott gerichtete Ausspruch: „[D]u hast uns auf dich hin geschaffen, und ruhelos ist unser Herz, bis es ruhet in dir! “34 Damit ist gemeint, dass dem Menschen eine Sehnsucht innewohnt, die innerweltlich nicht erfüllbar sei. Egal, was der Mensch diesseitig erreicht - selbst, wenn es Quasi-Unsterblichkeit sein sollte -, dieser inneren Unzufriedenheit könne der Mensch nur durch den Glauben entkommen. Christologisch und in der Geschichte des frühen Christentums ist in diesem Zusammenhang das Märtyrermotiv zentral: Nur im Blick auf ein ewiges Leben und unter der damit einhergehenden Relativierung irdischer Güter kann ein Mensch seine eigene Sterblichkeit vollständig bejahen, die Angst vor dem Tod überwinden und sein Leben für andere Menschen hingeben.

$\mathrm{Ob}$ es tatsächlich stimmt, dass alle innerweltlichen Güter ,nichtig“ sind, lässt sich rein argumentativ nicht eruieren. Möglicherweise ist nicht die Sterblichkeit das eigentliche Problem des Menschen, sondern das niemals erfüllbare Streben nach Unendlichkeit. Um Zufriedenheit zu erreichen, müsste dieser ,De-

\footnotetext{
33 Vgl. Ronald Cole-Turner. „Biotechnology and the Religion-Science Discussion“. The Oxford Handbook of Religion and Science. Hrsg. von Philip Clayton und Zachary Simpson. New York, NY: Oxford University Press, 2006. 929-944, S. 941.

34 Augustinus Aurelius. Des heiligen Augustinus Bekenntnisse. Übertragen und eingeleitet von Hubert Schiel. 6. Aufl. Freiburg: Herder, 1959, S. 1. Verbreitet ist auch die alternative Übersetzung ,Unruhig ist unser Herz, bis es ruht in dir‘.
} 
fekt' behoben werden. Einige philosophische Schulen (z. B. die antike Stoa) und sogar Religionen (wie der Buddhismus, aber auch einige Strömungen innerhalb des Christentums) gehen davon aus, dass der Mensch lernen muss, innere Seelenruhe zu erlangen und sein Glück nicht von Umständen abhängig machen soll, die nicht in seiner Macht liegen. So ist in Literatur und Künsten des Mittelalters und der Frühen Neuzeit das Motiv der ars moriendi - der Kunst zu sterben - bekannt; dieses lässt sich allerdings auf zweifache Weise deuten: Einerseits kann es sich auf die Fähigkeit beziehen, die eigene Vergänglichkeit und Sinnlosigkeit anzunehmen und den Charakter auf den Prozess des Sterbens (und nicht auf den Tod selbst) vorzubereiten. Andererseits, vor allem im christlichen Kontext, kann sich die ars moriendi auch auf die Fähigkeit beziehen, sein Leben auf die Existenz nach dem Tod, also auf das ewige Leben auszurichten. Der Mensch solle sich innerlich auf das Jüngste Gericht und die Gemeinschaft mit Gott vorbereiten, anstatt letztlich ,wertlose“ weltliche Güter anzustreben. ${ }^{35}$ Hier kann natürlich eingewandt werden, dass das Streben nach innerweltlichem Besitz und die Ausbildung eschatologisch relevanter Charaktereigenschaften nicht in einem notwendigen Widerspruch zueinander stehen. Doch lässt sich durchaus beobachten, dass es die Gefahr einer materialistisch und individualistisch orientierten Wohlstandsgesellschaft ist, die eschatologische Perspektive zu vernachlässigen.

Der Transhumanismus muss allerdings nicht automatisch auf der materialistischen Seite verortet werden. Die Agenda der Verbesserung des Menschen kann nämlich beide beschriebenen Formen aufgreifen: Man könnte einerseits versuchen, den Menschen dahingehend zu formen, dass bei ihm keine religiösen Gefühle, keine Verdrängung des Sterbens und keine ,falschen' Sehnsüchte wie die Sehnsucht nach Unendlichkeit mehr aufkommen. ${ }^{36}$ Medizinische, technischkybernetische und genetische Mittel könnten aber auch verwendet werden, um eine ,spirituelle Zielsetzung' voranzubringen und es dem Menschen zu erleichtern, entsprechende positive Charaktereigenschaften auszuprägen und Laster abzu-

35 Stark auf die christliche Deutung ausgerichtet sind zwei anonyme Texte, die im 15. Jahrhundert unter dem Titel ars moriendi als Reaktion auf die Pest-Epidemie entstanden sind. Vgl. „The Art of Dying Well“. Medieval Popular Religion, 1000-1500. A Reader. Hrsg. von John Shinners. London: Broadview, 1997. 525-535.

36 Charakterliche und spirituelle genetische Dispositionen lassen sich durch polygenic scoring statistisch immer besser voraussagen und in der Zukunft durch genetic enhancement wahrscheinlich auch signifikant beeinflussen. Vgl. Johannes Grössl. „Artificial Intelligence and Polygenic Scoring“. Artificial Intelligence. Reflections in Philosophy, Theology, and the Social Sciences. Hrsg. von Benedikt Göcke und Astrid Rosenthal-von der Pütten. Paderborn: Mentis, 2020. 347-360, S. 355-357. 
bauen. ${ }^{37}$ Beide Versuche würden aber in ihrer Umsetzung schnell in einen Konflikt mit der menschlichen Autonomie geraten, vor allem, wenn Individuen sich nicht mehr selbst für ein Enhancement entscheiden können (was vor allem bei genetischen Methoden mit Eingriff in die Keimbahn der Fall ist ${ }^{38}$ ) oder unter hohem gesellschaftlichen Druck stehen, bestimmte leistungsfördernde Enhancements, wie die Erhöhung von Intelligenz oder Verbesserung von Gesundheit, anzuwenden. ${ }^{39}$ Soll die menschliche Autonomie gewahrt werden, dürfen solche Eingriffe nur vorgenommen werden, wenn man davon ausgehen kann, dass der oder die Betroffene diesen Eingriffen rückwirkend zustimmen würde. ${ }^{40}$

\section{Ausblick}

Der Transhumanismus zielt darauf ab, die menschliche Sterblichkeit zu überwinden oder zumindest so lange wie möglich aufzuschieben. Mithilfe technologischer Verbesserungen soll den Menschen ein längeres, glücklicheres und gesünderes Leben ermöglicht werden. Doch gibt es einige Risiken der transhumanistischen Agenda, unter anderem, dass durch diese Verbesserungen andere Güter zerstört werden. Die Auseinandersetzung mit der eigenen Endlichkeit - die auch von zukünftigen Technologien nicht gänzlich überwunden werden kann - sowie die Auseinandersetzung mit der Vergänglichkeit anderer Menschen erhöht die Wahrscheinlichkeit, dass man sich mit Religion, Spiritualität und speziell der Frage nach einem ,Leben nach dem Tod' beschäftigt. Ohne diese Perspektive entfällt auch eine grundlegende Motivation zur Moralität: Immanuel Kant betrachtete die

37 Vgl. Jacob Shatzer. Transhumanism and the Image of God. Downers Grove, IL: Intervarsity Press, 2019. Roland Cole-Turner schreibt jedoch, dass der grundlegende Unterschied zwischen einem christlichen und einem nicht-christlichen Transhumanismus darin besteht, dass der christliche Glaube betont, dass Menschen nur zu ihrer Verwandlung beitragen, sie aber nicht aus eigener Kraft ausüben können; vgl. Cole-Turner 2006 (Anm. 33), S. 941-943.

38 Vgl. dazu Deutscher Ethikrat. Eingriffe in die menschliche Keimbahn. Stellungnahme vom 9. Mai 2019. https://www.ethikrat.org/fileadmin/Publikationen/Stellungnahmen/deutsch/stel lungnahme-eingriffe-in-die-menschliche-keimbahn.pdf (Zugriff am 09.01.2021), S. 39-40.

39 Vgl. ebd., S. 42 und S. 229-230.

40 Vgl. Benedikt Göcke. „Designobjekt Mensch?! Ein Diskursbeitrag über die Probleme und Chancen transhumanistischer Menschenoptimierung“. Designobjekt Mensch. Die Agenda des Transhumanismus auf dem Prüfstand. Hrsg. von dems. und Frank Meier-Hamidi. Freiburg: Herder, 2018. 117-152, S. 145: „[Ein] moralische[r] Transhumanismus [...] geht strikt davon aus, dass nur diejenigen Änderungen der biologischen Natur eines nicht-zustimmungsfähigen Erfahrungssubjektes vorgenommen werden dürfen, von denen mit hinreichender Gewissheit davon ausgegangen werden kann, dass ein autonomes und freies Erfahrungssubjekt sie a priori als moralisch relevante Verbesserung seines Wohlergehens selbst frei wählen würde.“ 
Existenz Gottes und die Existenz einer unsterblichen Seele als notwendige Bedingungen dafür, dass Glückswürdigkeit und Glückseligkeit zusammenfallen können, d. h., dass gute Menschen für ihre Taten belohnt und schlechte Menschen für ihre Taten bestraft werden - auch wenn dies erst zeitlich nach der irdischen Existenz geschieht. ${ }^{41}$

Obwohl es prima facie erstrebenswert ist, Menschen zu verbessern, müssen wir darüber diskutieren, welche menschlichen Eigenschaften wir verbessern möchten und ob manche Verbesserungen nicht auch gravierende Nebenwirkungen mit sich bringen. Das Vanitas-Motiv kann gerade heute dazu dienen, einem Technikoptimismus entgegenzutreten, und verhindern, dass innerweltliche Unsterblichkeit oder gar maximale innerweltliche Machtfülle - was Nietzsches ,Über-Mensch` anzeigt nicht ultimativ sinnstiftend sein können. Wenn die menschliche Natur wesentlich auf die Ausbildung von Autonomie mit der Möglichkeit zur freien Ausbildung eines tugendhaften Charakters ausgelegt ist, der eine ewige Gemeinschaft mit Gott ermöglicht, werden viele transhumanistische Ziele relativiert. Denn im Vergleich zu diesem Ziel wären alle weltlichen Dinge tatsächlich ,Nichtigkeit‘.

Sterblichkeit wird heute häufig als ein $\mathrm{zu}$ überwindendes und möglicherweise bald überwindbares Übel angesehen. Eine christliche Anthropologie tendiert hingegen dazu, sie als notwendige Bedingung für das Erreichen anderer Werte anzusehen und in eine eschatologische Perspektive einzubetten. Das Motiv der Vanitas, wie es im biblischen Buch Kohelet eingeführt wird, dient den Gläubigen als Erinnerung und den Nicht-Gläubigen als Warnung, dass alles Streben nach inner-weltlichen Gütern (ob egoistisch oder altruistisch motiviert) ohne Gottesperspektive letztlich sinnlos ist. Die Endlichkeit und Kontingenzbehaftetheit des Lebens führt zur Aufwertung von Entscheidungen, Charakterbildungsprozessen und der Relevanz der religiösen Heilsvorstellung als einer ewigen Gemeinschaft mit Gott.

41 Kant sah in diesem nur eschatologisch verwirklichbaren Zusammenfall von moralischer Glückswürdigkeit und Glückseligkeit das ,höchste Gut‘. Vgl. Otfried Höffe. Immanuel Kant. 7. Aufl. München: Beck, 2007, S. 256-258. Andererseits könnte man auch argumentieren, dass echte Moralität autonom sein muss und deswegen gerade der Wegfall der eschatologischen Perspektive wahrhaft selbstloses Handeln ermöglicht. Die christliche Tradition geht hier einen Mittelweg, indem sie fordert, dass bereits im irdischen Leben ein guter Charakter ausgeprägt werden muss, um der ewigen Verdammnis zu entgehen bzw. die Zeit im Purgatorium zu verkürzen; dies wird allerdings nicht als Belohnung oder Bestrafung angesehen. Das eschatologische Ziel darf nicht alleinige Motivation moralischen Handelns sein, weil dadurch das moralisch Gute und das aus egoistischer Sicht Erstrebenswerte zusammenfallen würden, was zur Aufhebung von Moralität führen würde. 
